\title{
Perfil imunogênico de vacinas inativadas contra agalaxia contagiosa em ovinos*
}

\section{Immunogenic profile of inactivated vaccines against contagious agalactia in sheep}

\author{
Aline Antas Cordeiro Cavalcanti, ${ }^{* *}$ Ana Claudia Campos, ${ }^{* *}$ Aline Guedes Mamede de Moraes, ${ }^{* *}$ Aldenir Cavalcanti de Lima, ${ }^{* *}$ \\ Marcia Almeida de Melo, ${ }^{* *}$ Edisio Oliveira de Azevedo***
}

\begin{abstract}
Resumo
Este trabalho teve como objetivo avaliar o perfil imunogênico em ovinos de três vacinas produzidas com linhagens brasileiras de Mycoplasma agalactiae. O perfil proteico do antígeno vacinal foi avaliado por SDS-PAGE e a imunogenicidade da vacina pela técnica de Western blot. A vacina foi inativada com formol, adsorvida em hidróxido de alumínio (Vacina 1), Montanide IMS-2215 (Vacina 2), Montanide Gel-01 (Vacina 3) e administradas em três doses. Entre a primeira e a segunda dose houve um intervalo de 21 dias, e entre a segunda e a terceira de 180 dias. O pool de soros de dez ovinos coletados nos períodos 0, 21, 35, 90, 150, 210, 270 e 360 dias pós-vacinação foram testados pela técnica de Western blot. A vacina 2 foi mais antigênica, com detecção de anticorpos 21 dias após a primeira dose. Para as vacinas 1 e 3, os anticorpos são verificados após 35 dias, com queda acentuada aos 90 dias; apenas anticorpos contra a proteína de 48 kDa apareceram após a terceira dose de forma discreta. Contra a vacina 2 , ainda persistiram anticorpos contra as proteínas de 48, 55 e 80 kDa nos períodos 90, 150 e 210, que aumentaram após a terceira dose. Conclui-se que a vacina 2 induziu a resposta humoral de forma estável contra proteínas de M. agalactiae.
\end{abstract}

Palavras-chave: antigenicidade, imunidade humoral, imunoblotting.

\begin{abstract}
This study aimed to evaluate the immunogenic profile in sheep of three vaccines produced with a field Brazilian strains of $M y c o p l a s m a$ agalactiae. The vaccine protein profile was evaluated by SDS-PAGE and vaccine immunogenicity by Western blot. The vaccine was inactivated with formaldehyde and adsorbed onto three different adjuvants: with aluminum hydroxide (Vaccine 1), Montanide IMS 2215 (Vaccine 2), and Gel Montanide-01 (Vaccine 3). The vaccine was administered in three doses. Between the first and second dose there was an interval of 21 days, and between the second and the third one of 180 days. A pool of ten sera collected in $0,21,35,90,150,210,270$ and 360 days after first vaccination were tested by Western blot techniques. The second vaccine was more antigenic with antibody detection 21 days after first dose. For both vaccines 1 and 3 , antibodies were present 35 days after first dose, with a significant drop at 90 days; only antibodies against $48 \mathrm{kDa}$ protein discreetly appeared after the third dose. Stimulation induced by vaccine 2 produced antibodies against 48, 55 and $80 \mathrm{kDa}$ proteins that persisted until 90 , 150 and 210 days after first dose, which rose again after third dose. It was concluded that the vaccine 2 induced stable humoral immunity against M. agalactiae proteins.
\end{abstract}

Keywords: antigenicity, humoral immunity, immunoblotting.

\section{Introdução}

O Mycoplasma agalactiae é o agente da agalaxia contagiosa $(\mathrm{AC})$, caracterizada por agalaxia, poliartrite e ceratoconjuntivite em caprinos e ovinos. A infecção causa perdas econômicas, principalmente em rebanhos leiteiros de caprinos e ovinos, devido aos surtos ou às infecções crônicas, com alta incidência de mastite subclínica (Gómez-Martín et al., 2013). A enfermidade tem distribuição mundial, sendo endêmica em Ásia, África e América (OIE, 2012).
Os primeiros casos da AC causada por M. agalactiae no Brasil ocorreram na Paraíba, disseminando-se para os Estados de Pernambuco e Rio Grande do Norte (Nascimento et al., 2002; Azevedo et al., 2006). Desde então, tem sido uma das principais preocupações dos caprinocultores devido aos sérios prejuízos econômicos.

O M. agalactiae possui diversidade antigênica de superfície devido a variações de fases na expressão de lipoproteínas imunodominantes denominadas de Vpmas (Variable proteins of

${ }^{*}$ Recebido em 17 de setembro de 2017 e aceito em 25 de setembro de 2018.

**Universidade Federal de Campina Grande, Departamento de Medicina Veterinária, Patos. Paraíba, Brasil.

***Universidade Federal de Sergipe, Departamento de Medicina Veterinária do Sertão, Nossa Senhora da Glória, Sergipe, Brasil.

Autor para correspondência: aline.antas@hotmail.com. 
M. agalactiae), contribuindo para os mecanismos de patogênese e evasão do sistema imune do hospedeiro (Bergonier et al., 1996; Flitman-Tene et al., 2000; Glew et al., 2000). Esta diversidade é observada em amostras isoladas em diferentes espécies, caprinos e ovinos, e em regiões geográficas distintas (Solsona et al., 1996). Os diferentes isolados apresentam bandas proteicas com 18 a 135 kD (Solsona et al., 1996; Tola et al., 1997). Amostras brasileiras de M. agalactiae, isoladas de caprino, apresentaram bandas protéicas de 30 a $135 \mathrm{kD}$, com imunogenicidade para as de 135, 55 e 48 kD (Campos, 2012).

As proteínas P55, P48, P40 e P30 são descritas como imunodominantes (Tola et al., 1997; Rosati et al., 2000; Fleury et al., 2001; Fleury et al., 2002; Santona et al., 2002) e vacinas tem sido testadas com o objetivo de evitar a infecção e eliminação do micoplasma (Greco et al., 2002; De La Fe et al., 2007a; 2007b). Recentemente, no Brasil, foram avaliadas, em caprinos e ovinos, vacinas inativadas constituída de $M$. agalactiae isolado de caprinos. Os melhores resultados foram obtidos com a vacina adsorvida com adjuvante oleoso (Campos, 2012).

A partir dos primeiros estudos que demonstraram o potencial das vacinas constituídas de $M$. agalactiae isoladas no Nordeste do país, o objetivo deste trabalho foi avaliar o perfil antigênico, em ovinos, das vacinas quando adsorvidas com adjuvante oleoso e aquoso.

\section{Material e Métodos}

As vacinas foram preparadas a partir da amostra de $M$. agalactiae (BrPB3.03) previamente caracterizada por Campos (2012), cultivada durante 96 horas em caldo Hayflick modificado, $\mathrm{pH} 7.8$, enriquecido com $20 \%$ de soro equino inativado a $37^{\circ} \mathrm{C}$ em microaerofilia. O cultivo foi centrifugado a $3800 \mathrm{~g}$ durante uma hora e ressuspenso em tampão salino fosfato [PBS, 137 $\mathrm{mM} \mathrm{NaCl}, 2.7 \mathrm{mM} \mathrm{KCl}, 10.2 \mathrm{mM} \mathrm{Na}_{2} \mathrm{HPO}_{4}, 1.8 \mathrm{mM} \mathrm{KH}_{2} \mathrm{PO}_{4}, \mathrm{pH}$ 7.6]. A suspensão bacteriana foi ajustada para uma concentração proteica pré-inativação de $5 \mathrm{mg} /$ dose da vacina e inativadas com formaldeído a $0,4 \%(\mathrm{v} / \mathrm{v})$ por 24 horas a $37^{\circ} \mathrm{C}$.

A suspensão inativada foi adsorvida sob agitação mecânica e constante durante três horas a temperatura ambiente, com o respectivo adjuvante: a) Vacina 1: Gel de hidróxido de alumínio na concentração de $6 \mathrm{mg} / \mathrm{mL}$ de vacina (adjuvante aquoso); b) Vacina 2: Montanide IMS 2215 VG PR (Seppic Inc.), na concentração de 25\% (v/v) (adjuvante oleoso); c) Vacina 3: Montanide Gel 01 PR (Seppic Inc.), a 5\% (v/v) (adjuvante aquoso) (Campos, 2012). As vacinas foram estocadas a $4^{\circ} \mathrm{C}$

Quarenta ovinos sem padrão racial definido, machos e fêmeas, com idade variando de 6 a 48 meses, foram distribuídos proporcionalmente em três grupos vacinais e um controle, com dez animais cada. Todos os animais foram negativos no teste de ELISA indireto para M. agalactiae (Campos et al., 2009).

A vacinação foi realizada em duas doses de $2 \mathrm{~mL}$ administradas por via subcutânea na região da axila direita e esquerda, previamente tricotomizadas, com intervalo de 21 dias entre as doses. Uma terceira dose foi administrada 180 dias após a segunda vacinação. O grupo controle recebeu o mesmo protocolo de vacinação utilizando PBS estéril como inóculo, como descrito por Campos (2012).
Os soros dos ovinos foram coletados nos dias $0,21,35,90$, 150, 210, 270 e 360 dias após a $1^{a}$ vacinação.

No Western blot (WB), a amostra de M. agalactiae (BrPB3.03), cultivada em meio Hayflick modificado, foi centrifugada a $12000 \mathrm{~g}$ por 20 minutos e o pellet ressuspenso em PBS, pH 7.6. A concentração protéica foi determinada por espectrofotometria (BioPhotometer Plus, Eppendorf, Alemanha) utilizando um filtro de $280 \mathrm{~nm}$. A amostra foi misturada em tampão de lise [500 mM Tris/HCl pH 6.8, 4.6\% (p/v) SDS, 20\% (v/v) glicerol, 10\% (v/v) 2-mercaptoetanol e $0.004 \%$ azul de bromofenol] e fervida por 5 minutos. Posteriormente, $15 \mu \mathrm{g}$ de proteínas de $M$. agalactiae foram separadas por SDS-PAGE 12\% (LAEMMLI, 1970). A eletroforese foi realizada a $250 \mathrm{~V}$ e $50 \mathrm{~mA}$ por gel durante 40 minutos. O gel de poliacrilamida foi corado em solução de Comassie blue [0.005\% (p/v) azul brilhante de Coomassie R-250, $7 \%(\mathrm{v} / \mathrm{v})$ ácido acético, 25\% (v/v) metanol].

As proteínas separadas por SDS-PAGE foram transferidas para membrana de nitrocelulose $0.45 \mu \mathrm{m}$ a $8 \mathrm{~V}$ e $700 \mathrm{~mA}$ por 70 minutos. As membranas foram bloqueadas em solução de bloqueio [PBS pH 7.6; 2\% (p/v) leite em pó desnatado (LPD)] por 30 minutos a $37^{\circ} \mathrm{C}$, e em seguida lavadas três vezes em PBS pH 7.6 por 5 minutos.

A membrana sensibilizada foi cortada em tiras e imersa no pool dos soros dos ovinos vacinados diluído 1:50 e nos controles positivo e negativo diluídos $1: 200$ e incubadas a $37^{\circ} \mathrm{C}$ por 45 minutos. O controle negativo do WB constituiu de um pool de soros de cinco caprinos não imunizados e negativos para $M$. agalactiae e o controle positivo de um pool de soros de cinco animais com sinais clínicos de agalaxia contagiosa, ambos os controles foram testados previamente pelo ELISA indireto (Campos et al., 2009).

Após incubação, as tiras foram lavadas três vezes por 5 minutos a temperatura ambiente com PBS e em seguida incubadas com conjugado proteína G peroxidase (Sigma-Aldrich) diluído 1:9000. As tiras foram novamente submetidas a lavagem e incubadas em solução de revelação [0.1 M tampão citrato $\mathrm{pH} 5.0,0.3 \mathrm{mg} /$ mL 3'-3'-5'-5'-tetrametilbenzidina (TMB) e $0.38 \%$ peróxido de hidrogênio]. Após cinco minutos, a reação foi interrompida com água destilada.

\section{Resultados e Discussão}

As vacinas foram capazes de induzir a produção de anticorpos IgG anti- $M$. agalactiae, entretanto as vacinas 1 e 3 foram menos antigênicas, apenas estimulando a resposta humoral após a segunda dose (tira 3, Figura 1).

Os adjuvantes interferem na resposta imune em relação ao antígeno, além da possibilidade de apresentar reação inflamatória na área de aplicação da vacina. De acordo com os dados do imunoblotting, a vacina 2 estimulou uma maior produção de anticorpos em relação as outras vacinas em todos os períodos, confirmando o estudo realizado por Campos (2012). A maior eficiência da vacina 2 deve-se ao tipo de adjuvante utilizado, resultados também obtidos por Buonavoglia et al. (2008; 2010), quando utilizaram adjuvantes oleosos na produção da vacina, e por De La Fe et al. (2007a, b) ao comparar o adjuvante oleoso com hidróxido de alumínio e com a saponina. 
A

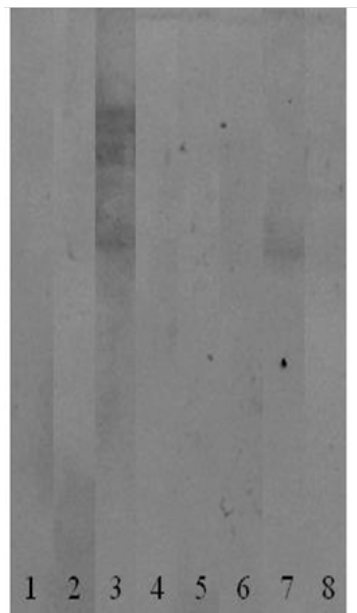

B

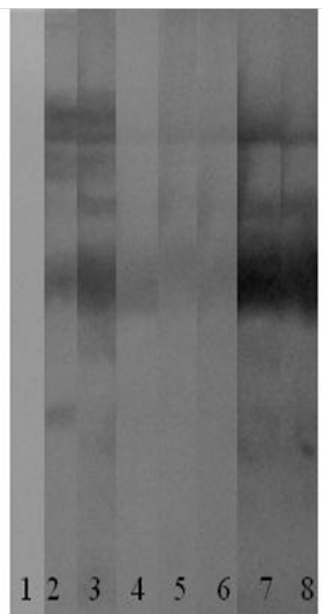

\section{C}

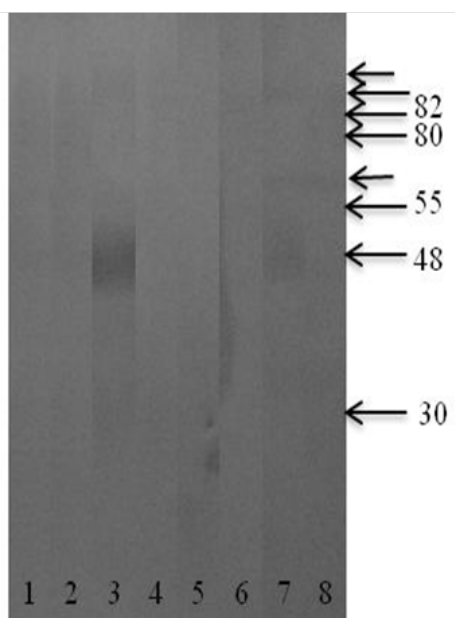

Figura 1: Imunoblotting de proteína total de $M$. agalactiae utilizando soros de ovinos vacinados com as vacinas experimentais 1 (A), 2 (B) e 3 (C). A numeração na fita corresponde aos dias após a $1^{\text {a }}$ dose da vacinação: $1: 1^{\text {a }}$ dose da vacina; $2: 21$ dias pós-vacinação; $3: 35$ dias pós-vacinação; $4: 90$ dias pósvacinação; 5: 150 dias pós-vacinação; 6: 210 dias pós-vacinação; 7: 270 dias pós-vacinação e 8: 360 dias pós-vacinação. Os pesos moleculares das proteínas em kDa estão indicados à direita.
Figura 1), e com aumento após o reforço nos dias 270 e 360 na vacina 2. Estes resultados são diferentes dos obtidos por Tola et al. (1997), onde os autores analisaram soros de animais naturalmente infectados até 30 dias do início dos sinais clínicos e observaram que a proteína de $55 \mathrm{kDa}$ foi fortemente imunogênica.

O estímulo para produção de anticorpos contra as proteínas de 82 e $80 \mathrm{kDa}$ ocorreu na vacina 2 após 21 dias da primeira vacinação e na vacina 1 apenas após a segunda dose. Noventa dias após a primeira dose já não se observam anticorpos contra a proteína de $82 \mathrm{kDa}$, mas há uma banda discreta para a de $80 \mathrm{kDa}$.

Com o reforço 180 dias após a segunda vacinação, surgem mais anticorpos contra a proteína de $80 \mathrm{kDa}$ (fitas 7 e 8, Figura 1). A P80 é uma das proteínas encontradas em $M$. agalactiae isoladas

Outro aspecto relevante em relação ao comportamento das vacinas é que na vacina 2 houve diferença quanto à imunogenicidade das proteínas. Nos períodos de 90, 150 e 210 dias após a primeira dose (fitas 4, 5 e 6, Figura 1), percebe-se a ausência de anticorpos contra a maioria das proteínas do micoplasma, mas com reaparecimento das bandas após o reforço, realizado seis meses após a segunda dose da vacina (fitas 7 e 8, Figura 1). Esta diminuição na produção de anticorpos é justificada por se tratar de uma vacina inativada, que estimula níveis de anticorpos mais baixos e menos persistentes, devendo ser repetida em períodos mais curtos (Leon Vizcaino et al., 1995; Buonavoglia et al., 1998). Para as vacinas 1 e 3 não houve aumento na eficiência da vacina, mesmo após o reforço, corroborando com Campos (2012).

Através do imunoblotting, observa-se que as proteínas com peso molecular de 82, 80, 55 e 48 kDa foram as mais imunogênicas, com imunodominância da última. Contra a proteína de 48 kDa houve produção de anticorpos mesmo nas vacinas 1 e 3 . Duas bandas acima de 55 kDa aparecem após segunda vacinação, mas não se mantêm após a terceira dose.

Campos (2012) relatou que a proteína de 48 kDa foi imunodominante para caprinos naturalmente infectados, corroborando com os resultados apresentados pelos animais vacinados neste estudo. A P48 é uma lipoproteína que estimula respostas imunes celular e humoral e com potencial para aplicação em diagnóstico (Rosati et al, 2000; Chessa et al., 2009).

Para a proteína de $55 \mathrm{kDa}$, a resposta humoral é observada a partir do dia 35, desaparecendo com 90 dias (fita 4, de caprinos na Espanha e de ovinos na Itália, infectados naturalmente (Tola et al., 1996; 1997; De La Fe et al., 2006) e foi reconhecida por soros ovinos na fase inicial da sintomatologia clínica (Tola et al., 1997).

Anticorpos contra a proteína de aproximadamente 82 kD não foi descrita como imunogênica por outros autores. No artigo de Tola et al. (1997) observa-se uma banda imunogênica em soros de ovinos com 30 dias após o início da sintomatologia clínica, logo acima da proteína de $80 \mathrm{kDa}$, mas os autores não tecem comentários.

Uma proteína de aproximadamente $30 \mathrm{kD}$ é visualizada na vacina 2 após 21 dias da primeira dose. Esta proteína foi detectada em diferentes amostras de $M$. agalactiae em estudos conduzidos por Tola et al. (1997) e De La Fe et al. (2006). Fleury et al. (2001) avaliaram a resposta humoral à P30 de ovelhas infectadas artificialmente com diferentes linhagens de $M$. agalactiae, e indicaram que a P30 é altamente imunogênica e induz uma resposta de anticorpos precoce que persiste durante, pelo menos, 2 meses após a infecção. O fato de não termos observado uma produção marcante de anticorpos anti-P30 pode ser em função de alterações na estrutura de proteína causadas durante a confecção da vacina.

\section{Conclusões}

A vacina 2 induziu a resposta humoral de forma estável contra proteínas de Mycoplasma agalactiae. 


\section{Referências}

AZEVEDO, E.O.; ALCÂNTARA, M.D.B.; NASCIMENTO, E.R.; TABOSA, I.M.; BARRETO, M.L.; ALMEIDA, J.F.; ARAÚJO, M.O.; RODRIGUES, A.R.O.; RIET-CORREA, F.; CASTRO, R.S. Contagious agalactia by Mycoplasma agalactiae in small ruminants in Brazil: first report. Brazilian Journal of Microbiology, v. 37, n. 4, p. 576-581, 2006.

BERGONIER, D.; DE SIMONE, F.; RUSSO, P.; SOLSONA, M.; LAMBERT, M. POUMARAT, F. Variable expression and geographic distribution of Mycoplasma agalactiae surface epitopes demonstrated with monoclonal antibodies. FEMS Microbiololy Letters, v. 143, n. 2-3, p. 159-65, 1996.

BUONAVOGLIA, D.; FASANELLA, A.; SAGAZIO, P.; TEMPESTA, M.; IOVANE, G.; BUONAVOGLIA, C. Persistence of antibodies to Mycoplasma agalactiae in vaccinated sheep. New Microbiologica, v. 21, n. 2, p. 209-212, 1998.

BUONAVOGLIA, D.; GRECO, G.; QUARANTA, V.; CORRENTE, M. MARTELLA, V.; DECARO, N. An oil-emulsion vaccine induces full-protection against Mycoplasma agalactiae infection in sheep. New Microbiologica, v. 31, n. 1, p. 117-123, 2008.

BUONAVOGLIA, D.; GRECO, G.; CORRENTE, M.; GRECO. M.F.; D'ABRAMO, M.; LATRONICO, F.; FASANELLA, A.; DECARO, N. Long-term immunogenicity and protection against Mycoplasma agalactiae induced by an oil adjuvant vaccine in sheep. Research in Veterinary Science, v. 88, n. 1, p. 16-19, 2010.

CAMPOS, A.C.; TELES, J.A.A.; AZEVEDO, E.O.; NASCIMENTO, E.R.; OLIVEIRA, M.M.M.; NASCIMENTO, S.A.; CASTRO, R.S. ELISA protein $G$ for the diagnosis of contagious agalactia in small ruminants. Small Ruminant Research, v. 84, n. 1-3, p. 70-75, 2009.

CAMPOS, A.C. Produção e avaliação de vacina contra agalaxia contagiosa. 2012. 75f. Tese (Doutorado em Ciência Veterinária) - Departamento de Medicina Veterinária, Universidade Federal Rural de Pernambuco, Recife, PE.

CHESSA, B.; PITTAU, M.; PURICELLI, M.; ZOBBA, R.; CORADDUZZA, E.; DALL'ARA, P.; ROSATI, S.; POLI, G.; ALBERTI, A. Genetic immunization with the immunodominant antigen P48 of Mycoplasma agalactiae stimulates a mixed adaptive immune response in BALBc mice. Research in Veterinary Science, v. 86, n. 3, p. 414-420, 2009.

DE LA FE, C.; ASSUNÇÃO, P.; ROSALES, R.S.; ANTUNES, T.; POVEDA, J.B. Characterization of protein and antigen variability among Mycoplasma mycoides subsp. mycoides (LC) and Mycoplasma agalactiae field strains by SDS-PAGE and immunoblotting. The Veterinary Journal, v. 171, n. 3, p. 532-538, 2006.

DE LA FE, C.; ASSUNÇÃO, P.; SAAVEDRA, P.; RAMÍREZ, A.; POVEDA, J.B. Field trial of a combined vaccine against caprine contagious agalactia: humoral immune response in lactating goats. The Veterinary Journal, v. 174, n. 3, p. 610-615, 2007a.

DE LA FE, C.; ASSUNÇÃO, P.; SAAVEDRA, P.; TOLA, S.; POVEDA, C.; POVEDA, J.B. Field trial of two dual vaccines against Mycoplasma agalactiae and Mycoplasma mycoides subsp. mycoides (large colony type) in goats. Vaccine, v. 25, p. 2340-2345, 2007b.

FLEURY, B.; BERGONIER, D.; BERTHELOT, X.; SCHLATTER, Y.; FREY, J.; VILEI, E.M. Characterization and Analysis of a Stable Serotype-Associated Membrane Protein (P30) of Mycoplasma agalactiae. Journal of Clinical Microbiology, v. 39, n. 8, p. 2814-2822, 2001.
FLEURY, B.; BERGONIER, D.; BERTHELOT, X.; PETERHANS, E.; FREY, J.; VILEI, E.M. Characterization of P40, a Cytadhesin of Mycoplasma agalactiae. Infetion and Immunity, v. 70, n. 10, p. 5612-5621, 2002.

FLITMAN-TENE, R.; LEVISOHN, S.; LYSNYANSKY, I. RAPOPORT, E.; YOGEV, D. A chromosomal region of Mycoplasma agalactiae containing vsp-related genes undergoes in vivo rearrangement in naturally infected animals. FEMS Microbiology Letters, v. 191, n. 2, p. 205-212, 2000.

GLEW, M.D.; PAPAZISI, L.; POUMARAT, F.; BERGONIER, D.; ROSENGARTEN, R.; CITTI, C. Characterization of a multigene family undergoing high-frequency DNA rearrangements and coding for abundant variable surface proteins in Mycoplasma agalactiae. Infection and Immunity, v. 68, n. 8, p. 4539-4548. 2000.

GÓMEZ-MARTíN, A.; AMORES, J.; PATERNA, A.; DE LA FE, C. Contagious agalactia due to Mycoplasma spp. in small dairy ruminants: Epidemiology and prospects for diagnosis and control. The Veterinary Journal, v. 198, n. 1, p. 48-56, 2013.

GRECO, G.; CORRENTE, M.; BUONAVOGLIA, D.; ALIBERTI, A.; FASANELLA, A. Inactivated vaccine induces protection against Mycoplasma agalactiae infection in sheep. New Microbiologica, v. 25, n. 1, p. 17-20, 2002.

LAEMMLI, U.K. Cleavage of structural proteins during the assembly of the head of bacteriophage T4. Nature, v. 227, p. 680 685, 1970.

LEÓN VIZCAÍNO, L.; GARRIDO ABELLÁN, F.; CUBERO PABLO, M.J.; PERALES, A. Immunoprophylaxis of caprine contagious agalactia due to Mycoplasma agalactiae with an inactivated vaccine. Veterinary Record, v. 137, n. 11, p. 266-269, 1995.

NASCIMENTO, E.R.; BARRETO, M.L.; PLATENIK, M.O.; TABOSA, I.M.; ALCÂNTARA, M.D.B. Contagious agalactia by Mycoplasma agalactiae in goats in Brazil. Etiologic study. In: CONGRESS OF THE INTERNATIONAL ORGANISATION FOR MYCOPLASMOLOGY (IOM), 14., 2002. Vienna, Austria. Proceedings...Vienna: [s.n.] 2002. p. 45-46.

OIE. Organização Mundial de Saúde Animal, 2012.

Disponível em: http://www.oie.int/wahis_2/public/wahid.php/ Diseaseinformation/Diseasetimelines. Acesso em 13 abr. 2013.

ROSATI, S.; ROBINO, P.; FADDA, M.; POZZI, S.; MANNELLI, A.; PITTAU, M. Expression and antigenic characterization of recombinant Mycoplasma agalactiae P48 major surface protein. Veterinary Microbiology, v. 71, n. 3-4, p. 201-210, 2000.

SANTONA, A.; CARTA, F.; FRAGHÍ, P.; TURRINI, F. Mapping antigenic sites of an immunodominant surface lipoprotein of Mycoplasma agalactiae, AvgC, with the us of synthetic peptides. Infection and Immunity, v. 70, n. 1, p. 171-176, 2002.

SOLSONA, A.; LAMBERT, M.; POUMARAT, F. Genomic, protein homogeneity and antigenic variability of Mycoplasma agalactiae. Veterinary Microbiology, v. 50, n. 1-2, p. 45-58, 1996

TOLA, S.; IDINI, G.; MANUNTA, D.; CASCIANO, I.; ROCCHIGIANI, A.M.; ANGIOI, A.; LEORI, G. Comparison of Mycoplasma agalactiae isolates by pulsed field gel electrophoresis, SDS-PAGE and immunoblotting. FEMS Microbiology Letters, v. 143, n.2-3, p. 259-265, 1996.

TOLA, S.; MANUNTA, D.; COCCO, M.; TURRINI, F.; ROCCHIGIANI, A.M.; IDINI, G.; ANGIOI, A.; LEORI, G Characterization of surface proteins of Mycoplasma agalactiae during natural infection. FEMS Microbiology Letters, v. 154, n. 2, p. 355-362, 1997. 